\title{
Interactions Between Growth Cones and Neurites Growing from Different Neural Tissues in Culture
}

\author{
Josef P. Kapfhammer and Jonathan A. Raper \\ Max-Planck-Institut für Entwicklungsbiologie, 7400 Tübingen, Federal Republic of Germany
}

\begin{abstract}
We have previously used retinal and sympathetic explants to show that growth cones recognize and retract from specific neurites in culture (Kapthammer et al., 1986). In an effort to determine the generality of this phenomenon and to see how many different neurite labels can be detected by it, we have studied interactions between individual growth cones and neurites extending from a variety of neural sources in vitro.

Using most of the possible pairings between sympathetic, ciliary, dorsal root ganglion (DRG), retinal, and diencephalic neurons, we have found that in most instances: (1) Growth cones do not retract from neurites originating from the same tissue; (2) retinal growth cones do not retract from diencephalic neurites; (3) sympathetic, ciliary, and DRG growth cones, with one possible exception, do not retract from sympathetic, ciliary, or DRG neurites; (4) retinal growth cones retract from sympathetic, ciliary, and DRG neurites; (5) sympathetic, ciliary, and DRG growth cones retract from retinal neurites; and (6) sympathetic growth cones retract from diencephalic neurites.
\end{abstract}

A simple hypothesis consistent with these results is that 2 labels exist-one associated with central neurites and another associated with peripheral neurites-and that peripheral growth cones are programmed to retract from the central label and central growth cones are programmed to retract from sympathetic, ciliary, and DRG neurites; (5) symevant to the separation of the CNS and PNS during development.

The interaction of growth cones with neurites in their immediate environment can play an important role in growth cone guidance (Raper et al., 1983, 1984; Bonhoeffer and Huf, 1985; Bastiani et al., 1986; Kuwada, 1986). Time-lapse cinematography has been used in the past to study the behavior of growth cones contacting neurites in simple tissue culture environments. Nakajima (1965) reported that chick growth cones extending in a plasma clot either cofasciculated on, crossed over, or retracted away from neurites in their path. Dunn (1971) explained the radial extension of neurites from dorsal root ganglia (DRG) grown in plasma clots by the contact inhibition of growth cones that contacted other neurites. In contrast, Wessells et al. (1980) reported that individual growth cones extending from chick

Received Sept. 16, 1986; revised Nov. 10, 1986; accepted Nov. 20, 1986.

We thank Susannah Chang and David Tonge for their criticism of the manuscript, Mike McKenna for helpful suggestions, and Barbara Grunewald for tech nical assistance. This work was supported by the Max-Planck-Gesellschaft. J.P.K received a stipend from the Studienstiftung des deutschen Volkes.

Correspondence should be addressed to Jonathan A. Raper, MPI Entwicklungsbiologie, Spemannstrasse 35, 7400 Tübingin, F.R.G.

Copyright (C) 1987 Society for Neuroscience 0270-6474/87/051595-06\$02.00/0
DRG or ciliary neurons growing on polylysine or collagen substrates cross other axons easily and without contact inhibition.

Using cocultures of retinal and sympathetic explants in a manner similar to that described by Bray et al. (1980), we have shown that sympathetic growth cones extending on laminin cross sympathetic neurites without inhibition. The same is true for retinal growth cones crossing retinal neurites. However, sympathetic growth cones retract on contact from retinal neurites, as do retinal growth cones from sympathetic neurites (Kapthammer et al., 1986). When a growth cone touches an incompatible neurite, its normal, flattened, motile morphology collapses and its trailing neurite retracts (Kapfhammer and Raper, 1987). A new growth cone is usually organized after a brief delay. These events-contact mediated paralysis, retraction, and recoverybear a strong resemblance to the contact inhibition of locomotion that occurs between many non-neuronal cells (Abercrombie, 1970).

These results cannot be explained by nonspecifc, purely passive mechanisms. Instead, they suggest that some form of specific cell-cell recognition triggers the collapse of growth cone motility. They imply that there exists at least one difference between sympathetic and retinal neurites that allows growth cones to distinguish between them. Also implicit is a complementary difference in retinal and sympathetic growth cones that causes them to react in opposite ways to the same stimulus.

These findings are consistent with the labeled pathways hypothesis, originally proposed to explain the navigational abilitics of middle and late-growing neurites in the CNS of invertebrates (Goodman et al., 1982; Raper et al., 1983). It predicts that (1) axons are specifically labeled and (2) growth cones are differentially programmed to prefer to grow in association with certain labels as opposed to others.

One object of this study was to determine if the growth cone retractions we described between retinal and sympathetic tissues are representative of a more general phenomenon, applicable to a wide range of growth cone-neurite pairings. If so, our second object was to determine how many different neurite labels can be detected in this way. To these ends, we tested most of the possible growth cone-neurite pairings of DRG, sympathetic ganglia, ciliary ganglia, and retinal tissues, as well as pairings of retinal or sympathetic growth cones with a population of early diencephalic neurites.

Wc found that, as a rulc, growth cones from the PNS do not retract from other PNS neurites but do retract from the limited selection of CNS neurites we tested. Retinal growth cones retract from PNS neurites but not from the CNS neurites we tested. These results tentatively suggest that the retraction phenomenon we have described reflects a general difference between central and peripheral neurites and growth cones. 


\section{Table 1. Culture media used for the different growth cone-neurite} combinations

Medium

Tissue combinations cultured

F12

Ret gc vs. Ret; Sym gc vs. Sym;

Ret gc vs. Sym; Ret ge vs. DRG;

DRG gc vs. DRG; Ret gc vs. DEC;

Sym gc vs. DRG; DRG gc vs. Sym;

Sym gc vs. Ret; DRG gc vs. Ret

1-d HCM $2 / 3$

+ 3-d HCM $1 / 3$

Cil gc vs. Cil; Cil gc vs. Sym;

F12 or $1-\mathrm{d} H C M$

Sym gc vs. Cil; Ret gc vs. Cil

Sym gc vs. DEC

F12 is the medium described by Kapfhammer et al (1986) $1-\mathrm{d} \mathrm{HCM}$ is $\mathrm{F} 12$ medium conditioned $1 \mathrm{~d}$ over heart cells (Kapthammer et al., 1987), and 3-C HCM is the same medium conditioned for $3 \mathrm{~d}$. Abbreviations: Ret, retina; $\mathrm{gc}$, growth cone; Sym, sympathetic; DRG, dorsal root ganglion; DEC, diencephalon; Cil, ciliary.

\section{Materials and Methods}

Explants. Embryonic day 6 (E6) retinal explants and E8 sympathetic explants were made as described earlier (Kapfhammer et al., 1986). DRGs were dissected from the lumbosacral region of E8 chick embryos and cut into halves or quarters before culturing. Ciliary ganglia were taken from E9 chick embryos and cut into halves. The diencephalon of E4 chick embryos was dissected and cut into pieces measuring approximately $250 \times 250 \mu \mathrm{m}$ before culturing. We do not know the identitiy of the very long neurites $(>1000 \mu \mathrm{m})$ that grow from these explants, although it is possible that they normally contribute to the very early developing, descending thalamotegmental and/or medial longitudinal pathways (Windle and Austin, 1936). All explants were positioned 2$4 \mathrm{~mm}$ apart and briefly pressed against the substrate to aid in their attachment (Kapfhammer et al., 1986).

Cultures. All explants were grown on laminin-coated glass coverslips (Kapfhammer et al., 1986). Most explant pairs were cultured in F12 medium with $5 \%$ chick serum, $5 \%$ fetal calf serum, and supplemented as in Kapfhammer et al. (1986). Some tissue combinations were cultured in the same medium conditioned by a monolayer of heart cells (Kapfhammer and Raper, 1987). It is difficult to obtain optimal growth from all the tissues in the same medium. Sympathetic explants grow best in medium conditioned for at least 1 day $(1 \mathrm{~d} \mathrm{HCM})$, ciliary explants in medium conditioned $3 \mathrm{~d}(3 \mathrm{~d} \mathrm{HCM})$, and retinas in unconditioned medium or medium conditioned no more than 1 day. The medium we used for each pairing (Table 1) was chosen as a healthy compromise between the competing requirements of each of the 2 explants.

Recordings and analysis. Video recordings were taken between 20 and $72 \mathrm{hr}$ of culture time. For the tissue combinations involving diencephalic explants, a piece of diencephalic tissue was cultured for $48 \mathrm{hr}$ until the time when significant fiber outgrowth had begun. Then, a second explant was positioned nearby and viedo recordings were made between 20 and $48 \mathrm{hr}$ thereafter.

The video system, the selection of recordings, the identification of the involved growth cones and neurites, and the methods of analysis of the recordings were the same as described in Kapfhammer et al. (1986). In brief, situations were chosen at random in which growth cones and neurites could be traced back to their explant of origin. Only cases in which growth cones approached neurites at an angle of between $60^{\circ}$ and $120^{\circ}$ were analyzed. Video recordings were made with a $25 \times$ lens and at a time-lapse factor of $125 \times$. The paths of growth cones were traced from the screen as the videos were replayed and converted into plots relating the distances between growth cones and neurites as a function of time. The numbers for retractions, crossings, and delays were extracted from these plots according to our previous definitions (Kapfhammer et al., 1986).

\section{Results}

Like pairs

We tested the bchavior of growth cones that were confronted with neurites from the same tissues. Figure 1 illustrates the distances between growth cones and neurites ( $y$-axis) as a function of time ( $x$-axis). These values are positive as the growth cones approach neurites, zero as they cross them, and negative thereafter (see Kapfhammer et al., 1986). Figure $1 A$ shows representative confrontations between a retinal growth cone and a retinal neurite (Fig. $1 A-1$ ), a sympathetic growth cone and a sympathetic neurite (Fig. $1 A-2$ ), a DRG growth cone and a DRG neurite (Fig. $1 A-3$ ), and a ciliary growth cone and a ciliary neurite (Fig. $1 A-4$ ). In each of these pairings growth cones are hardly influenced by the neurites in their paths. They generally cross like neurites without retracting, and are consequently delayed little or not at all.

\section{Unlike CNS pairs and unlike PNS pairs}

The same pattern holds for 1 pairing between 2 CNS tissues, and all but one of the pairings between 3 PNS tissues. Retinal growth cones cross diencephalic neurites without retraction or delay (Fig. 1B-1). Retraction generally does not occur between sympathetic growth cones confronting DRG neurites (Fig. $1 B$ 2), DRG growth cones confronting sympathetic neurites (Fig. $1 B-3$ ), or ciliary growth cones confronting sympathetic neurites (Fig. $1 B-4$ ). In all of these CNS vs. CNS or PNS vs. PNS pairings, growth cones behave as if they were meeting neurites from their own tissue of origin. The only exception to this pattern is that a majority of sympathetic growth cones retract (Fig. $1 B-5)$ and are delayed when crossing ciliary neurites.

\section{Retinal growth cones versus PNS neurites}

The only type of CNS growth cone we used in this study was from retinal explants, since in our cultures the extension of growth cones from other CNS tissues we tested was not consistent enough to be analyzed. Retinal growth cones cross PNS neurites with greater difficulty than CNS neurites. Retinal growth cones meeting sympathetic (Fig. 1C-1), DRG (Fig. 1C-2), or ciliary neurites (Fig. $1 C$-3) usually retract on contact. Although their advance is delayed, they generally cross peripheral neurites upon readvancing once or twice. These results are in sharp contrast to the lack of retraction or delay shown by retinal growth cones crossing retinal (Fig. $1 A-1$ ) or diencephalic neurites (Fig. $1 B-1)$.

\section{PNS growth cones vS. CNS neurites}

Growth cones from PNS tissues generally retract from retinal neurites. Sympathetic (Fig. $1 D-1$ ), DRG (Fig. 1D-2), and ciliary (Fig. 1D-3) growth cones usually retract on contact. Their advance is greatly delayed, and they are frequently unable to cross retinal neurites at all. The same pattern holds for sympathetic growth cones that meet diencephalic neurites (Fig. 1D-4), although sympathetic growth cones cross diencephalic neurites more frequently than retinal neurites (Table 2 ).

\section{Quantitative results}

We analyzed between 16 and 53 growth cone-neurite interactions for each tissue pair we studied. The majority of growth cones behaved like the representative examples given in Figure 1 for each tissue pair. There were always some growth cones, however, that behaved differently. The complete quantitative data for all the growth cone neurite interactions we analyzed are given in Table 2 . Listed for every tissue combination are the number of interactions analyzed, the precentage of growth cones that retracted from neurites, the percentage of growth cones that crossed neurites, and the percentage of growth cones 

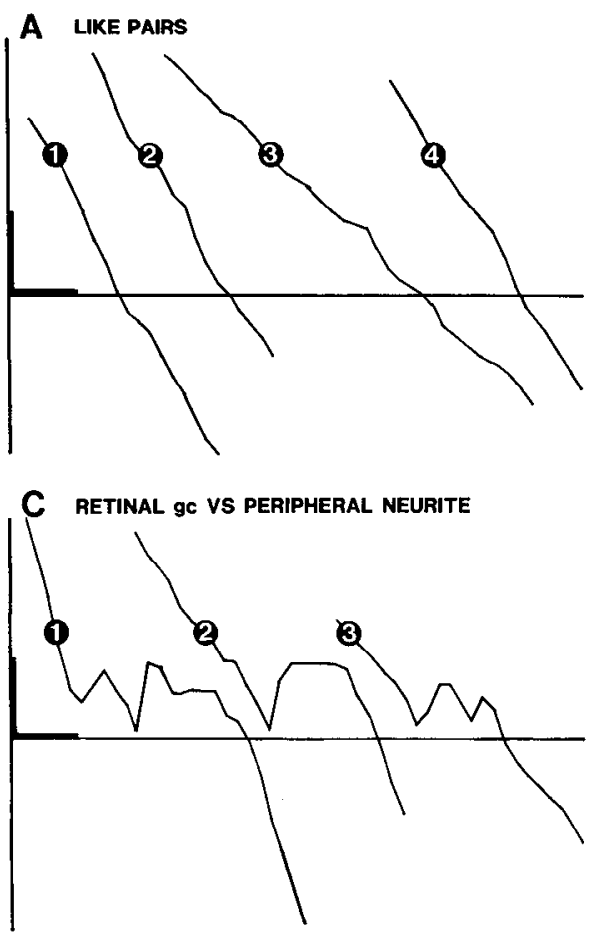
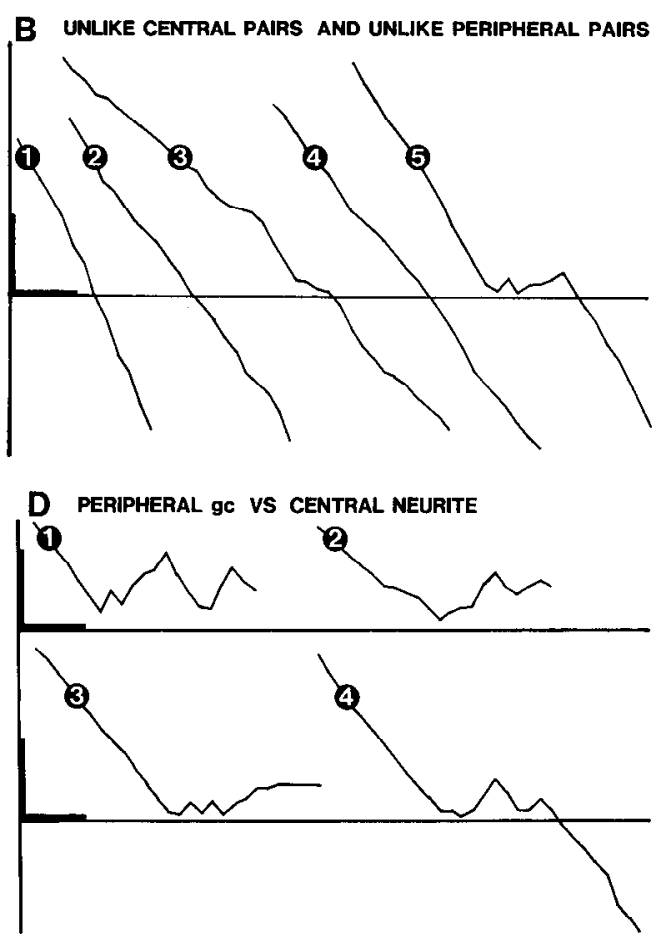

Figure 1. Selection of plots from representative growth cone neurite encounters. Time is shown on the $x$-axis, distance between growth cone and neurite on the $y$-axis. A growth cone crosses a neurite when $y=0$. Abbreviations: Ret, retina; Sym, sympathetic; Cil, ciliary; DRG, dorsal root ganglion; DEC, diencephalon; gc, growth cone. Calibrations (thickened regions of axes), 30 min, $50 \mu \mathrm{m}$. $A$, Interactions between growth cones and neurites from the same tissues: 1 , Ret gc vs. Ret; 2 , Sym gc vs. Sym; 3, DRG gc vs. DRG; 4, Cil gc vs. Cil. $B$, Interactions between growth cones and neurites from unlike CNS tissues and between growth cones and neurites from unlike PNS tissues: 1, Ret gc vs. DEC; 2 , Sym gc vs. DRG; 3 , DRG gc vs. Sym; 4 , Cil gc vs. Sym; 5 , Sym gc vs. Cil. $C$, Interactions between retinal growth cones and neurites from PNS tissues: 1, Ret gc vs. Sym; 2, Ret gc vs. DRG; 3 , Ret gc vs Cil. $D$, Interactions between growth cones from PNS tissues and neurites from CNS tissues: 1, Sym gc vs. Ret; 2 , DRG gc vs. Ret; 3, Cil gc vs. Ret; 4 , Sym ge vs. DEC. that were delayed for longer than 10 min by neurites.

It can be seen that retinal and ciliary growth cones are less often delayed by like neurites than are sympathetic and DRG growth cones. In the latter 2 pairings, there is a significant minority of instances in which retraction or delay occurs.

Retinal growth cones generally do not retract nor are they significantly delayed when meeting retinal or diencephalic neurites. The same is true for DRG growth cones meeting DRG or sympathetic neurites, ciliary growth cones meeting ciliary or sympathetic neurites, or sympathetic growth cones meeting sympathetic or DRG neurites. Sympathetic growth cones are more likely to retract from ciliary neurites than they are from sympathetic or DRG neurites.

The percentages of retractions and delays are very high for all combinations of retinal growth cones confronting PNS neurites or PNS growth cones confronting CNS neurites. However, retinal growth cones are more likely to cross PNS neurites than PNS growth cones are to cross CNS neurites.

These results are reflected by the distribution of delay times for each pairing (Fig. 2). These distributions are always skewed towards shorter delay times when like growth cones meet like neurites (Fig. 2: $A, E, J, N$ ), when retinal growth cones meet diencephalic neurites (Fig. $2 B$ ), or when PNS growth cones meet other PNS neurites (Fig. 2: $F, K, O$ ). In contrast, delay times are skewed to the right whenever retinal growth cones meet PNS neurites (Fig. 2: $C, G, L$ ) or when PNS growth cones meet CNS neurites (Fig. 2: $D, H, M, Q$ ). The distributions of delay times are remarkably similar for those pairings in which retraction generally does not occur (Fig. 2: first and second columns). The distributions are also similar for pairings in which retraction generally occurs (Fig. 2: third and fourth columns).

A summary of all our results is given in Table 3. Pluses mark those growth cone-neurite combinations in which significant retractions and delays are found. Minuses mark those growth cone-neurite combinations in which no significant retractions or delays occur.

\section{Discussion}

In 2 previous papers we have shown that retinal growth cones retract on contact from sympathetic neurites but not from retinal neurites, while sympathetic growth cones retract on contact from retinal neurites but not from sympathetic neurites (Kapfhammer

Table 2. Numerical results for all growth cone-neurite combinations

\begin{tabular}{llrrr} 
Combination & $n$ & $\% \mathrm{R}$ & $\% \mathrm{X}$ & $\% \mathrm{D}$ \\
\hline Like pairs & & & & \\
Ret gc vs. Ret & 44 & 2 & 100 & 9 \\
Sym gc vs. Sym & 29 & 28 & 93 & 45 \\
DRG gc vs. DRG & 17 & 12 & 100 & 47 \\
Cil gc vs. Cil & 21 & 5 & 100 & 14
\end{tabular}

Unlike central pairs and unlike peripheral pairs

$\begin{array}{lccrr}\text { Ret gc vs. DEC } & 39 & 8 & 97 & 15 \\ \text { Sym gc vs. DRG } & 18 & 11 & 100 & 22 \\ \text { DRG gc vs. Sym } & 16 & 13 & 100 & 44 \\ \text { Cil gc vs. Sym } & 31 & 10 & 100 & 16 \\ \text { Sym gc vs. Cil } & 49 & 55 & 96 & 61 \\ \text { Retinal gc vs. peripheral neurite } & & & \\ \text { Ret gc vs. Sym } & 38 & 79 & 68 & 84 \\ \text { Ret gc vs. DRG } & 32 & 66 & 72 & 69 \\ \text { Ret gc vs. Cil } & 22 & 91 & 77 & 86 \\ \text { Peripheral gc vs. central neurite } & & & \\ \text { Sym gc vs. Ret } & 24 & 96 & 29 & 96 \\ \text { DRG gc vs. Ret } & 33 & 91 & 42 & 97 \\ \text { Cil gc vs. Ret } & 21 & 95 & 48 & 100 \\ \text { Sym gc vs. DEC } & 53 & 85 & 62 & 89\end{array}$

For each combination is shown the number of growth conc-neurite interactions analyzed $(n)$, the percentage of cases where growth cones retracted from neurites $(\% \mathrm{R})$, the percentage of cases where growth cones crossed neurites $(\% \mathrm{X})$, and the percentage of cases where growth cones were delayed by neurites for more than $10 \mathrm{~min}(\% \mathrm{D})$. The growth cone-neurite combinations are arranged in the same order as in Figure 1. 


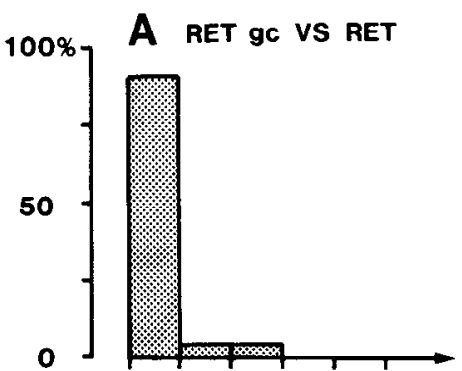

B RET gC vS DEC
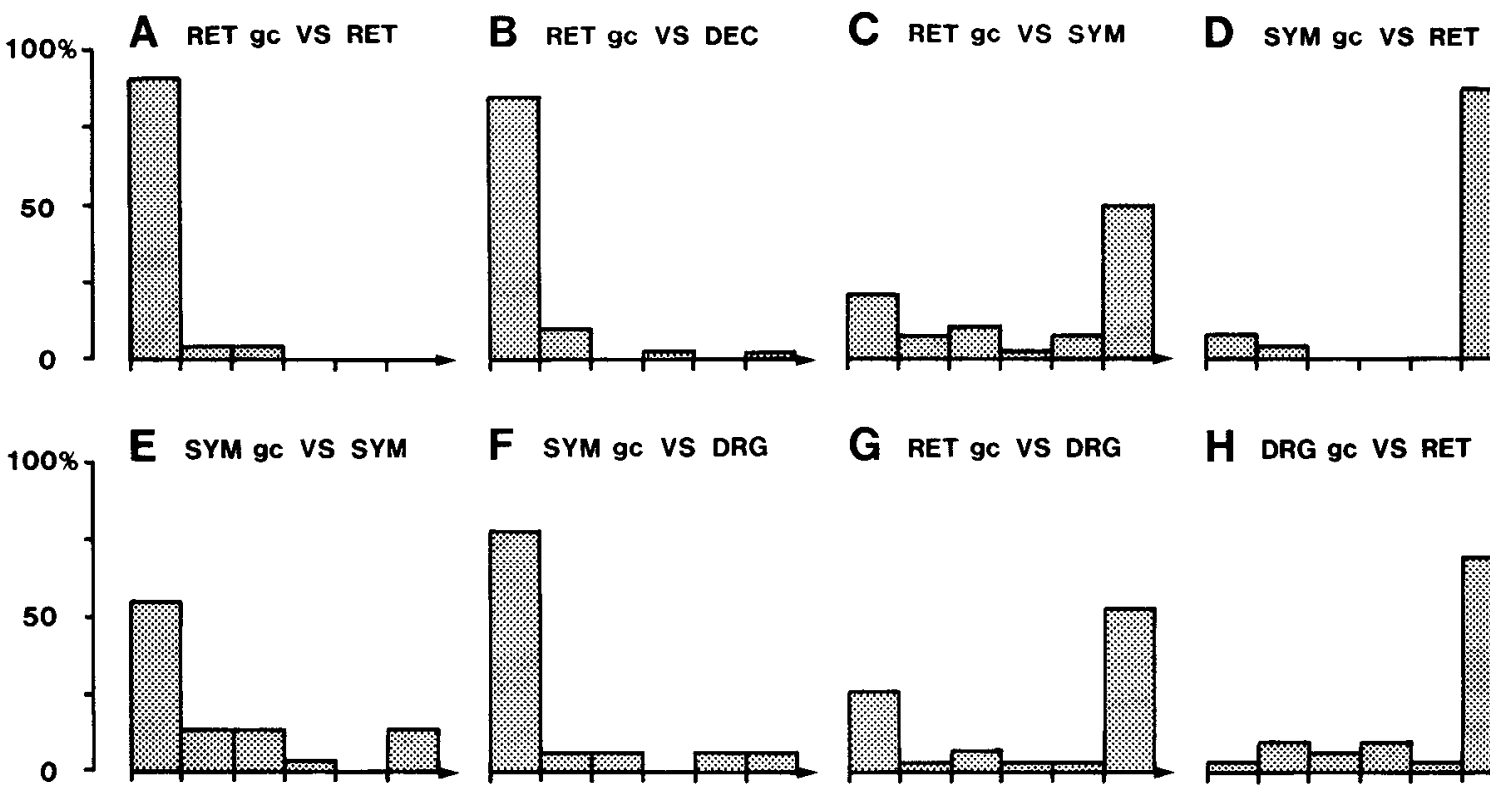

F SYM ge VS DRG
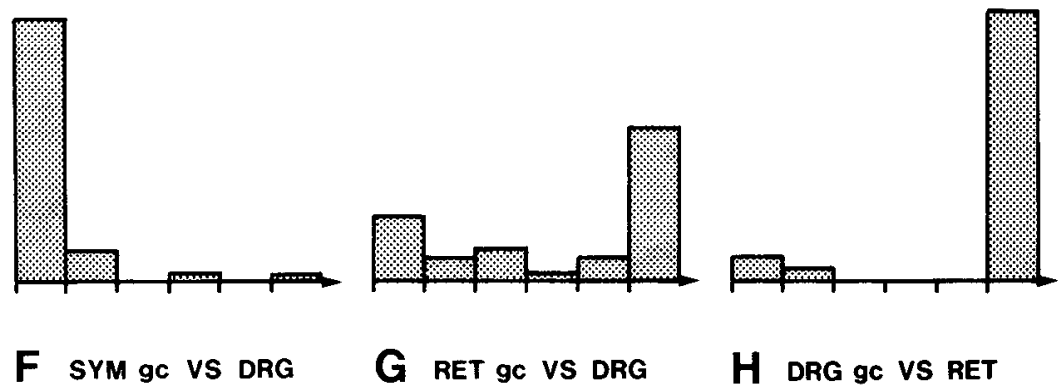

H DRG ge VS RET

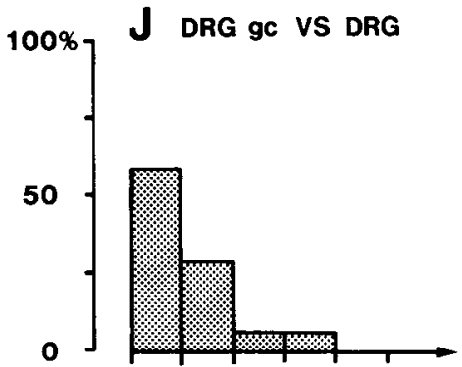

K DRG ge vs SYM

L RET ge VS ClL
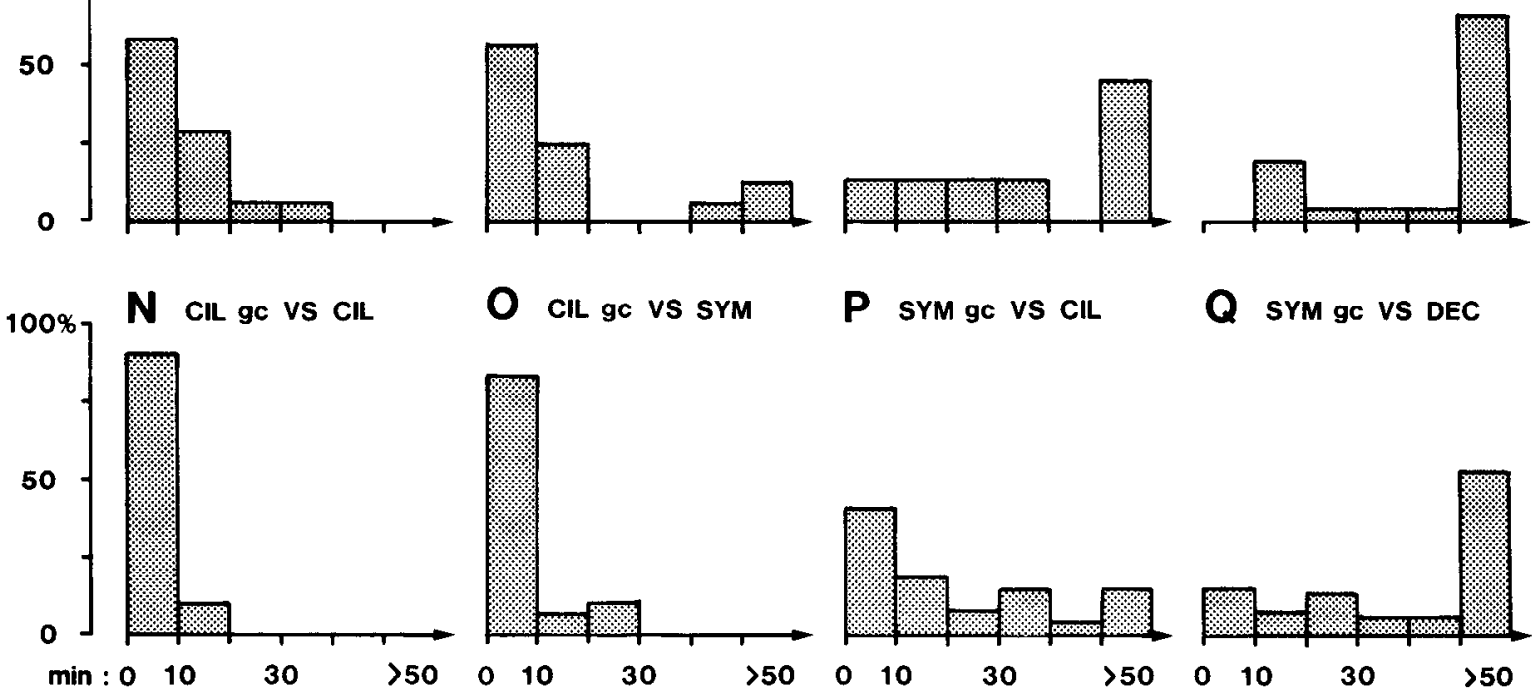

P sYM ge vs CIL

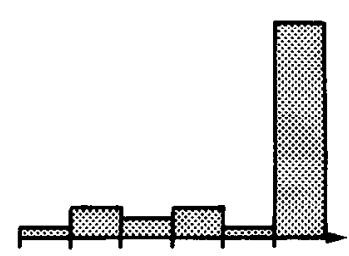

M cIl gC VS RET
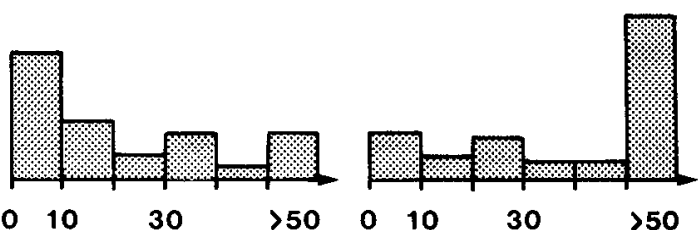

Figure 2. Distributions of delay times for various growth cone-neurite combinations. Each histogram shows the percentage of delay times that fell into each of the $10 \mathrm{~min}$ intervals indicated on the bottom. The last bin represents all delay times $>50$ min. The number of cases analyzed for each combination is given in Table 2. Abbreviations as in legend to Figure 1.

et al., 1986; Kapfhammer and Raper, 1987). These results imply that sympathetic and retinal neurites are differentially labeled and that sympathetic and retinal growth cones behave differently when confronted with the same labels. In an effort to determine the generality of this phenomenon and to determine how many different axonal labcls might be detected in this manner, we have now extended our observations to include interactions between a variety of growth cones and neurites.

Thus far, our results suggest that growth cones generally do not retract from neurites arising from the same neural tissue. Within this generalization, some differences may be found. Retinal growth cones retract from retinal neurites and ciliary growth cones retract from ciliary neurites less often than DRG growth cones retract from DRG neurites or sympathetic growth cones retract from sympathetic neurites. One possible explanation for these differences is that more frequent retractions within a tissue type reflect a greater heterogeneity of labels within that tissue.

Our results also suggest that peripheral growth cones generally do not retract from other peripheral neurites. This pattern holds true for all but 1 pairing, in which sympathetic growth cones meet ciliary neurites. Sympathetic growth cones retract $25 \%$ of the time from sympathetic neurites, $50 \%$ of the time from ciliary neurites, and more than $80 \%$ of the time from retinal or diencephalic neurites. The retraction of sympathetic growth cones from ciliary neurites is therefore relatively weak. The sympathetic-ciliary pairing is also unusual in that it is the only combination in which we observed asymmetric interactions between 2 tissues. Ciliary growth cones respond very similarly to both 


\begin{tabular}{|c|c|c|c|c|c|}
\hline & Ret & DEC & Sym & DRG & Cil \\
\hline Ret gc & - & - & + & + & + \\
\hline$\overline{\text { Sym gc }}$ & + & + & $\overline{-}$ & - & \pm \\
\hline DRG gc & + & n.d. & - & - & n.d. \\
\hline Cil gc & + & n.d. & - & n.d. & - \\
\hline
\end{tabular}

Growth cones are given at left, neurites above. Combinations in which the majority of growth cones cross neurites without being inhibited are marked with a minus (no inhibition); combinations in which the majority of growth cones retract and are delayed by neurites are marked with a plus (inhibition); ambiguous cases are marked with both symbols $( \pm)$; and combinations that were not tested are marked "n.d." (not determined). The lines separate CNS from PNS tissues.

ciliary neurites and sympathetic neurites, while sympathetic growth cones are more likely to retract from ciliary as opposed to sympathetic neurites.

Our results further suggest that peripheral growth cones retract from central neurites and that retinal neurites retract from peripheral neurites.

The simplest rule consistent with these results is that peripheral growth cones retract from central neurites, central growth cones retract from peripheral neurites, peripheral growth concs do not retract from peripheral neurites, and central growth cones do not retract from central neurites. This pattern could be generated by a very small number of labels. The minimal requirements would be that central and peripheral neurites have at least one difference between them and that central and peripheral growth cones have a complementary difference responsible for their conflicting behavior when faced with the same cues.

One particularly straightforward hypothesis is that there exists a "central" axonal label and a "peripheral" axonal label: Only peripheral growth cones read and retract in response to the central label, and only central growth cones read and retract in response to the peripheral label. Relevant to this notion are the findings of Cohen and Selvendran (1981) and Vulliamy et al. (1981), who demonstrated the existence of CNS- and PNS-specific cell surface antigens.

The possibility that therc is a division into central and peripheral neurite labels must be considered a tentative hypothesis, subject to disproof based on data from a wider range of tissue combinations.

An alternative hypothesis is that we detected incompatibilities between central and peripheral tissues merely because, of those we tested, they are the least alike. The peripheral tissues we used in this study represent a broad spectrum of neural crest-derived phenotypes, including sensory, sympathetic, and parasympathetic ganglia. It might be useful to include placode-derived tissues in future studies. Our results are also necessarily based on a very narrow range of central tissues, which should be augmented in the future.

The specific repulsive effects produced by labels divided between the CNS and PNS might partially explain the observation that long projecting axons rarely extend for great distances in both the CNS and PNS. One exception, the simultaneous extension of DRG axon segments in the dorsal spinal cord and in the periphery, could be explained if the segments possess separate peripheral and central labels. This presumed segregation of peripheral and central labels within 1 neuron might, in turn, explain why nearly $50 \%$ of our cultured DRG growth cones were delayed by DRG neurites. However, this line of reasoning does
Table 4. A simple labeling scheme consistent with the present data

\begin{tabular}{llll} 
Tissue & Labels & Receptivity & Tissue avoided \\
\hline Sym & $\mathrm{P}$ & $\mathrm{C}^{\prime}, \mathrm{X}^{\prime}$ & Ret, DEC, Cil \\
DRG & $\mathrm{P}$ & $\mathrm{C}^{\prime}$ & $\underline{\text { Ret, }}$ DEC \\
Cil & $\mathrm{P}, \mathrm{X}$ & $\mathrm{C}^{\prime}$ & $\underline{\text { Ret, }}, \mathrm{DEC}$ \\
Ret & $\mathrm{C}$ & $\mathrm{P}^{\prime}$ & $\underline{\text { Sym }}$, DRG, Cil \\
DEC & $\mathrm{C}$ & $?$ & $?$
\end{tabular}

Each tissue is listed in the leftmost column. A hypothetical assignment of neurite labels is made in the second column. Growth cone receptivity is indicated in the third column. If a growth cone's receptivity matches a label, retraction is assumed to occur. Predicted retractions are listed in the last column, retractions actually observed in this study are underlined. Abbreviations: tissues as in Table 1; $\mathrm{P}$, peripheral; $\mathrm{C}$, central; $\mathrm{X}$, a third label; $\mathrm{P}^{\prime}, \mathrm{C}^{\prime}$, and $\mathrm{X}^{\prime}$ represent the corresponding receptivities.

not help to explain the same observation for sympathetic-sympathetic interactions. A possibility of more general interest is suggested by another exceptional case. Rohon-Beard cells are primary sensory neurons in amphibia and fish. They extend their axons in the CNS (Kuwada, 1986) and PNS (Roberts and Hayes, 1977) early enough to avoid the interference of other axon types. They may be able to grow long distances in both the CNS and PNS by virtue of their early start. The same strategy might also apply to DRG neurites. Even if they possess an exclusively "peripheral" label, they enter the cord early enough to avoid the interference of central axons (Ramon y Cajal, 1972; E. B. Grunewald and J. A. Raper, unpublished observations) and thereby establish their own exclusive tract for later-arriving DRG axons to grow in. Finally, a growth cone capable of growing in association with any label could be generated by removing or inactivating the receptors on the growth cone that read the label.

We obtained only 1 result hinting at labels that do more than differentiate central and peripheral processes, specifically, the retraction of sympathetic growth cones from ciliary neurites. If the intermediate level of sympathetic-ciliary retractions we observed implies an incompatibility between sympathetic growth cones and ciliary neurites, and keeping in mind that there is no corresponding incompatibility between ciliary growth cones and sympathetic neurites, the presence of as few as 3 separate axonal labels would comfortably explain all of our results (Table 4).

Similar additional incompatibilities between axon pairs could, in theory, coexist independently with a more general antipathy between central and peripheral neurites. Since it is in the CNS that neurite-neurite interactions can be expected to play their greatest role in growth cone guidance, it may be that additional labels will be found when a more extensive range of central pairings is possible.

It is somewhat disappointing that such a small number of putative labels can explain all our results. By implication, the labeled pathway hypothesis envisions a reasonably wide spectrum of axonal labels. That they were not forthcoming in this study might suggest that (1) if they exist, they are positive rather than negative in their effects; (2) a wide variety of labels is more likely to be found on central than on peripheral neurites; (3) the appropriate labels are not expressed in our culture conditions; or (4) labels are more likely to be detected between neurites known to interact in vivo, as opposed to the unphysiological pairings necessarily used in this study.

Growth cone motility has been shown to be specifically inhibited by particular non-neuronal cells (Nuttall and Zinsmeister, 1983; Verna, 1985), by a freely diffusible neurotransmitter 
(Haydon et al., 1985), and on contact with specific axons. Specific negative interactions like these could play an important role in growth cone guidance. Our findings tentatively suggest that negative growth cone-neurite interactions could play a role in the separation of the CNS and PNS.

\section{References}

Abercrombie, M. (1970) Contact inhibition in tissue culture. In Vitro 6: $128-142$.

Bastiani, M. J., S. du Lac, and C. S. Goodman (1986) Guidance of neuronal growth cones in the grasshopper embryo. I. Recognition of a specific axonal pathway by the pCC neuron. J. Neurosci. 6: 35183531 .

Bonhoeffer, F., and J. Huf (1985) Position-dependent properties of retinal axons and their growth cones. Nature 315: 409-410.

Bray, D., P. Wood, and R. P. Bunge (1980) Selective fasciculation of nerve fibers in culture. Exp. Cell. Res. 130: 241-250.

Cohen, J., and S. Y. Selvendran (1981) A neuronal cell surface antigen is found in the CNS but not in peripheral neurons. Nature 291: 421423.

Dunn, G. H. (1971) Mutual contact inhibition of extension of chick sensory nerve fibers in vitro. J. Comp. Neurol. 143: 491-508.

Goodman, C. S., J. A. Raper, R. Ho, and S. Chang (1982) Pathfinding by neuronal growth cones during grasshopper embryogenesis. Symp. Soc. Dev. Biol. 40: 275-316.

Haydon, P. G., D. B. Cobb, and S. B. Kater (1984) Serotonin selectively inhibits growth cone motility and synaptogenesis of specific identified neurons. Science 226: 561-564.

Kapfhammer, J. P., and J. A. Raper (1987) Collapse of growth cone structure on contact with specific neurites in culture. J. Neurosci. 7 : 201-212.

Kapfhammer, J. P., B. E. Grunewald, and J. A. Raper (1986) The selective inhibition of growth cone extension by specific neurites in culture. J. Neurosci. 6: 2527-2534.
Kuwada, J. Y. (1986) Cell recognition by neuronal growth cones in a simple vertebrate embryo. Science 233: 740-746.

Nakajima, S. (1965) Selectivity in fasciculation of nerve fibers in vitro. J. Comp. Neurol. 125: 193-204.

Nuttall, R. P., and P. P. Zinsmeister (1983) Differential response to contact during embryonic nerve-nonnerve cell interactions. Cell Motil. 3: 307-320.

Ramon y Cajal, S. (1972) Histogenese de la Moelle epiniere et des ganglion rachidiens. In Histologie du Systeme Nerveux de l'Homme et des Vertebres, Vol. 1, Instituto Ramon Y Cajal, Madrid.

Raper, J. A., M. J. Bastiani, and C. S. Goodman (1983) Pathfinding by neuronal growth cones in grasshopper embryos: II. Selective fasciculation onto specific axonal pathways. J. Neurosci. 3: 31-41.

Raper, J. A., M. J. Bastiani, and C. S. Goodman (1984) Pathfinding by neuronal growth cones in grasshopper embryos: IV. The effects of ablating the $A$ and $P$ axons upon the behavior of the $G$ growth cone. J. Neurosci. 4: 2329-2345.

Roberts, A., and B. P. Hayes (1977) The anatomy and function of free nerve endings in an amphibian skin system. Proc. R. Soc. London [Biol.] 296: 415-429.

Verna, J.-M. (1985) In vitro analysis of interactions between sensory neurons and skin: Evidence for selective innervation of dermis and epidermis. J. Embryol. Exp. Morphol. 86: 53-70.

Vulliamy, T., S. Rattray, and R. Mirsky (1981) Cell surface antigen distinguishes sensory and autonomic peripheral neurons from central neurons. Nature 291: 418-420.

Wessells, N. K., P. C. Letourneau, R. P. Nuttall, M. Luduena-Anderson, and J. M. Geiduschek (1980) Responses to cell contacts between growth cones, neurites and ganglionic non-neuronal cells. J. Neurocytol. 9: 647-664.

Windle, W. F., and M. F. Austin (1936) Neurofibrillar development in the central nervous system of chick embryos up to 5 days' incubation. J. Comp. Neurol. 63: 431-463. 